\title{
The variance-covariance method: microdosimetry in time-varying low dose-rate radiation fields
}

\author{
J. Breckow, A. Wenning, H. Roos, and A.M. Kellerer \\ Institut für Medizinische Strahlenkunde der Universität, Versbacher Strasse 5, \\ D-8700 Würzburg, Federal Republic of Germany
}

Received February 1, 1988 / Accepted in revised form April 20, 1988

\begin{abstract}
Summary. The variance-covariance method is employed at low doses and in radiation fields of low dose rates from an ${ }^{241} \mathrm{Am}(4 \mathrm{nGy} / \mathrm{s})$ and a ${ }^{90} \mathrm{Sr}$ $(300 \mathrm{nGy} / \mathrm{s})$ source. The preliminary applications and results illustrate some of the potential of the method, and show that the dose average of lineal energy or energy imparted can be determined over a wide range of doses and dose rates. The dose averages obtained with the variance-covariance method in time-varying fields, for which the conventional variance method is not suitable, agree well with results obtained under the condition of constant dose rate. The results are compared to data obtained in terms of the conventional single-event measurements. The method has evident advantages, such as facility and speed of measurement.
\end{abstract}

\section{Introduction}

The Variance-Covariance Method (Kellerer and Rossi 1984) permits the determination of the dose mean energy imparted, $\bar{\varepsilon}_{D}$, the specific energy, $\bar{z}_{D}$, or the lineal energy, $\bar{y}_{D}$, in radiation fields of fluctuating intensity. Two detectors $A$ and $B$, are exposed to the same radiation field and register synchronously the energy imparted within each of the detectors. From a series of sampling intervals one can determine the variance of the signals for each detector, and the covariance between the signals of the two detectors. The dose average of the energy imparted per energy deposition event (ICRU 1980, 1983) is:

$$
\begin{aligned}
& \bar{\varepsilon}_{D, A}=\left(V\left(\varepsilon_{A}\right)-C\left(\varepsilon_{A}, \varepsilon_{B}\right)\right) \cdot \bar{\varepsilon}_{A} \\
& \bar{\varepsilon}_{D, B}=\left(V\left(\varepsilon_{B}\right)-C\left(\varepsilon_{A}, \varepsilon_{B}\right)\right) \cdot \bar{\varepsilon}_{B}
\end{aligned}
$$

where $\varepsilon_{A}, \varepsilon_{B}$ : energies imparted in volumes $A$ and $B$ during sampling intervals; $V\left(\varepsilon_{A}\right), V\left(\varepsilon_{B}\right)$ : relative variance of $\varepsilon_{A}$ or $\varepsilon_{B} ; C\left(\varepsilon_{A}, \varepsilon_{B}\right)$ : relative covariance of $\varepsilon_{A}$ and $\varepsilon_{B}$. 
The magnitude of $V(\varepsilon)$ is determined not only by the fluctuations of energy imparted (stochastic component), but also by any variation of absorbed dose per sampling interval (dose-rate fluctuations). The objective of the microdosimetric measurements is the determination of the inherent statistical fluctuations of energy imparted, and one must, therefore, correct for the unwanted but frequently unavoidable dose-rate fluctuations. This correction is effected by subtracting the relative covariance, as indicated in (1). Part of the covariance can also be due to other fluctuations that affect both detectors equally, for example pressure or voltage drifts which change the gain; (1) accounts for the possible influence of such factors, too. The conventional variance method is applicable only in those cases where the fluctuations can be avoided, or can be kept sufficiently small to be accounted for by approximative corrections.

The mean values, $\bar{\varepsilon}_{A}$ and $\bar{\varepsilon}_{B}$, of the specific energies, the mean values, $\varepsilon_{A}^{2}$ and $\overline{\varepsilon_{B}^{2}}$, of their squares, and the mean value, $\overline{\varepsilon_{A} \cdot \varepsilon_{B}}$, of their products are obtained from a series of consecutive sampling intervals. In terms of the averages from $N(\gg 1)$ sampling intervals one obtains the estimate that corresponds to (1):

$$
\bar{\varepsilon}_{D, A}=\sum_{j=1}^{N} \varepsilon_{A j}^{2} / \sum_{j=1}^{N} \varepsilon_{A j}-\sum_{j=1}^{N} \varepsilon_{A j} \cdot \varepsilon_{B j} / \sum_{j=1}^{N} \varepsilon_{B j} .
$$

In the following, preliminary investigations with the variance-covariance method are reported. A comparison is made between the dose average event size obtained from the conventional single-event analysis and from variance-covariance measurements. Certain basic features of the instrumentation are examined, in order to assess the range of applicability of the new method, with respect to dose and dose rate. Numerical results are presented which were obtained in measurements at low dose rates from ${ }^{241} \mathrm{Am}$ and ${ }^{90} \mathrm{Sr}$ sources.

\section{Materials and methods}

Two identical cylindrical counters, as shown in the diagram of Fig. 1, are mounted in close proximity to each other. The preliminary studies were concerned merely with methodological aspects. Accordingly, there was no need in this study, to employ tissue equivalent counters. The detector shells are stain-

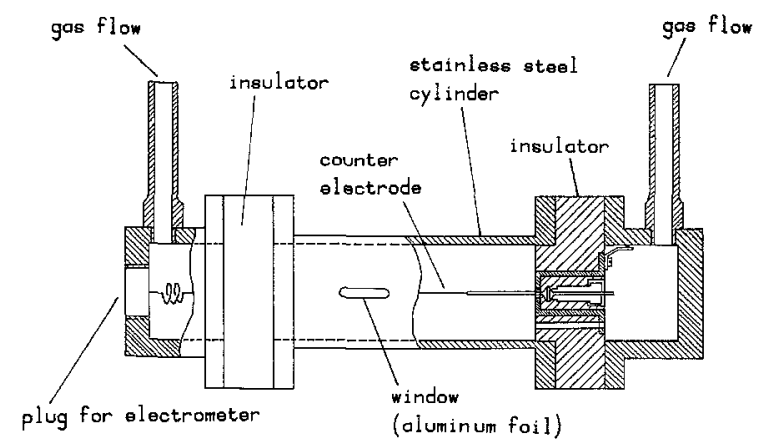

Fig. 1. Proportional-counter design utilized in the twindetector device 

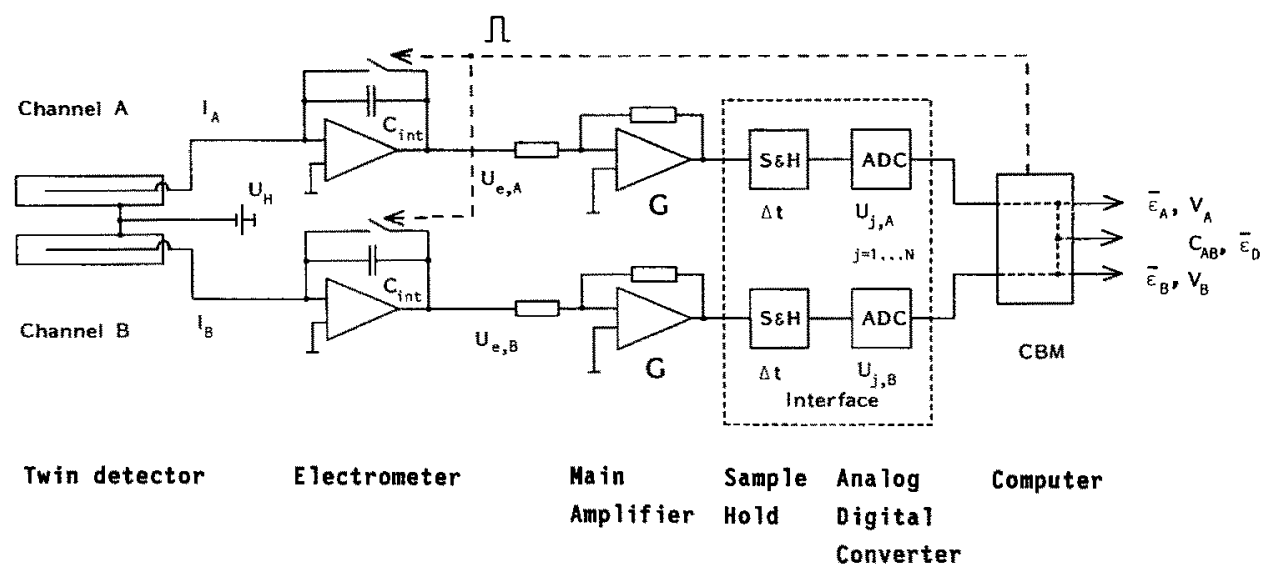

Fig. 2. Block diagram of the electronic circuit for the Variance-Covariance Method. Details are described in the text

less steel cylinders (outer diameter: $24 \mathrm{~mm}$, inner diameter: $20 \mathrm{~mm}$, length: $50 \mathrm{~mm}$ ) insulated against the central electrodes (diameter: $20 \mu \mathrm{m}$, effective length: $20 \mathrm{~mm}$ ) by polyethylene disks. The counters are linked to the same system of methane-gas flow with a gas pressure of $10.6 \mathrm{kPa}$ corresponding to a density of $7.5 \cdot 10^{-5} \mathrm{~g} / \mathrm{cm}^{3}$. Thus, the diameter and the height of the simulated cylindrical volume (density: $1 \mathrm{~g} / \mathrm{cm}^{3}$ ) are $1.5 \mu \mathrm{m}$. Windows on the detectors (aluminum foil, $20 \mu \mathrm{m}$ ) permit measurements even with low energy test sources. They have the effect that the fluence is not uniform throughout the sensitive volume of the counters. For the present explorative study this is of little concern; however, it needs to be noted since the microdosimetric variables $\varepsilon, z$, or $y$ are usually related to small sites over which the fluence is constant. In the present discussion reference is made primarily to the energy imparted, $\varepsilon$, although the considerations apply equally to $z$ and $y$.

Electrode voltage and gas pressure are adjusted to ensure proportional mode of operation. The detector current, $I$, is proportional to the dose rate, $\dot{D}$, and to the gas multiplication. The currents are measured by electrometers (Keithley Instruments, Operational Amplifier 301) working as charge amplifiers, i.e., as current integrators (Fig. 2). The possible integration times are determined by the current (dose rate), the integration capacitance, $C_{\mathrm{int}}$, and the voltage range of the electrometer $(U=0$ to $14 \mathrm{~V})$. The capacitance, $C_{\text {int }}$ can be varied down to about $30 \mathrm{pF}$, but a typical value in the actual measurements was $250 \mathrm{pF}$. The capacitors are periodically discharged by computer controlled relays. The discharge-time constant is of the order of $100 \mathrm{~ms}$.

For improved voltage resolution (see below) the output signal, $U_{e}(t)$, of each electrometer is passed through a main amplifier (gain $G=2$ to 995 ) which in turn feeds a Sample-and-Hold Device (SH). The SH-signal sampling at times $t_{j}=j \cdot \Delta t$ is controlled by an internal clock with frequencies, $f=1 / \Delta t$, of $1 \mathrm{~Hz}$, $10 \mathrm{~Hz}, 100 \mathrm{~Hz}$, or $1 \mathrm{kHz}$. The successive voltage values are digitized by an Analog-Digital-Converter (ADC, integral part of the Interface Commodore Model 4270). This results in a set of values, $\left\{U_{j}\right\}$ for one sampling series. The data 
transfer is controlled by a microcomputer (CBM 8032). The 12 bit digitizing of the voltages in the ADC (dynamic range $U=0$ to $10 \mathrm{~V}$ ) results in a resolution of $10 \mathrm{~V} / 4096 / \mathrm{G} \approx 2 \mathrm{mV} / \mathrm{G}$.

With the present interfacing, the data transfer is limited by the time required for transmitting one pair of data to the microcomputer and by the time for the AD conversion. The limiting rate of synchronous transfer of data pairs is $4 \mathrm{kHz}$; in actuality a maximum sampling frequency of $1 \mathrm{kHz}$ and up to $N=500$ pairs of data (=number of sampling intervals) per sampling series are utilized. The voltage increment for a sampling interval of duration $\Delta t$ is proportional to the energy imparted, $\varepsilon_{j}$ :

$$
U\left(t_{j+1}\right)-U\left(t_{j}\right)=\Delta U_{j}=\frac{G}{C_{\mathrm{int}}} \cdot \int_{t_{j}}^{t_{j+t}} I(t) \cdot \mathrm{d} t \sim \varepsilon_{j}
$$

with

$$
t_{j+1}-t_{j}=\Delta t=1 / f
$$

$C_{\text {int }}$ : integration capacitance; $G$ : gain of the main amplifier.

The average voltage increment per sampling interval is proportional to the mean energy imparted and is obtained from the formula:

$$
\overline{\Delta U}=\frac{1}{N} \sum_{j=1}^{N} \Delta U_{j} \sim \bar{\varepsilon} .
$$

The estimates for the mean of the squares and the mean of the products are analogous:

$$
\begin{aligned}
& \overline{\Delta U^{2}} \quad=\frac{1}{N} \sum_{j=1}^{N} \Delta U_{j}^{2} \sim \overline{\varepsilon^{2}}, \\
& \overline{\Delta U_{A} \cdot \Delta U_{B}}=\frac{1}{N} \sum_{j=1}^{N} \Delta U_{A j} \cdot \Delta U_{B j} \sim \overline{\varepsilon_{A} \cdot \varepsilon_{B}} .
\end{aligned}
$$

A sampling series, which contains, as stated, a maximum number of $N=500$ data pairs, can be obtained within one single charge integration process. Alternatively, and in order to achieve better resolution, one can subdivide a sampling series into $m$ sampling cycles each covering the total dynamic range of the ADCs $(10 \mathrm{~V})$; this permits the choice of substantially higher gains. Each cycle (containing $k=N / m$ data) is terminated by discharging the integration capacitors.

Figure 3 gives an example for this procedure in terms of the chains of values for a complete sampling series in one detector; the values were obtained from the current integration and voltage sampling in a constant ${ }^{241} \mathrm{Am}$ radiation field (cf. Sect. 3.1). In this particular case $m=6$ sampling cycles, each of $k=80$ data pairs, result in a total number of $N=480$ data pairs for the entire sampling series.

In a further step of data processing, one eliminates the voltage gaps which occur between successive cycles due to the discharge of the capacitors; thus, 
Fig. 3. Upper panel: Current integration and signal sampling in a constant ${ }^{241} \mathrm{Am}$ radiation field for one of the detectors. The sampling frequency is $f=1 \mathrm{~Hz}$ (气sampling interval $1 \mathrm{~s}$ ). The number of sampling cycles is $m=6$; each covers a range of $10 \mathrm{~V}$ and contains $k=80$ sample points per cycle corresponding to a total sample number $N=480$ and a total time of $480 \mathrm{~s}$. Lower panels: The corresponding voltage increments $\Delta U(t)$ from the detectors $A$ and $B$
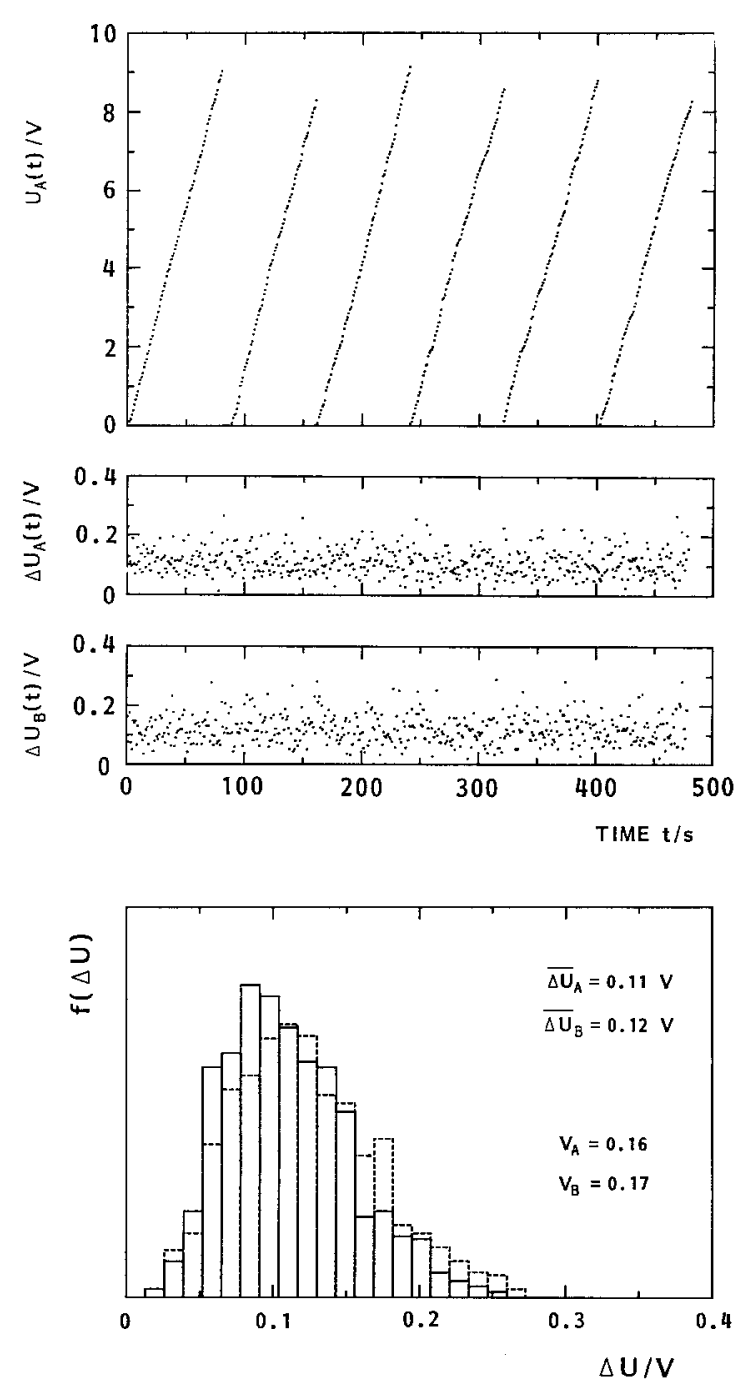

Fig. 4. Frequency distributions of the voltage increments $\Delta U_{j}$ from the detectors $A$ (solid line) and $B$ (dashed line); the data are those from Fig. 3. The mean values, $\overline{\Delta U}$, and the relative variances, $V$, are indicated

the signals for adjacent cycles are joined. In this way the total dynamic range for $N$ values is expanded to $m \cdot 10 \mathrm{~V}$. For the entire sampling series one then determines the voltage differences $\Delta U_{j}$, which are indicated, for the present example, in the lower panels of Fig. 3. The distributions of the increments $\Delta U_{j}$ of the two detectors are represented in Fig. 4. The mean values, $\overline{\Delta U}$, and the relative variances, $V$, are also given in the figure. Figure 5 gives the corresponding correlation diagram of the paired values $\Delta U_{A j}$ and $\Delta U_{B j}$ in the sampling series. As to be expected for a measurement under constant dose rate, the relative covariance is small $\left(C=3 \cdot 10^{-3}\right)$, i.e., the values are essentially uncorrelated.

Energy calibration has been performed with various characteristic $\mathrm{x}$-ray energies. Six energies between $1.5 \mathrm{keV}$ and $5 \mathrm{keV}$ were used to calibrate pulse heights in single-event measurements in terms of the energy imparted to the detector. 


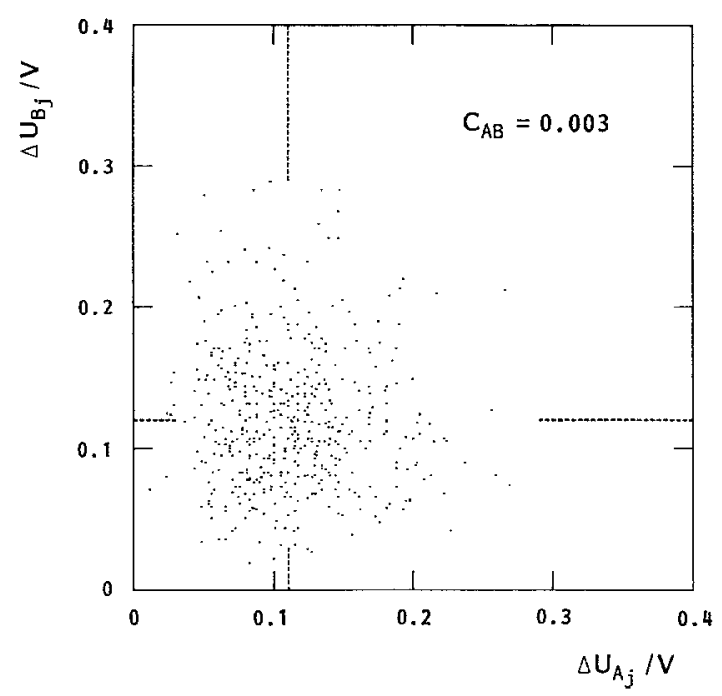

Fig. 5. Correlation diagram of the voltage increments from the detectors $A$ and $B$; the data are those from Fig. 3. The dashed lines represent the mean values $\overline{\Delta U}$. The relative covariance, $C_{A B}$, is indicated

\section{Results}

To assess the applicability of the variance-covariance method with the prototype experimental set-up, it was necessary to perform additional measurements with the conventional single-event technique, and also to perform measurements with no radiation field present. These matters are described in the subsequent sections. Data will be presented which were obtained with an ${ }^{241} \mathrm{Am}$ and a ${ }^{90} \mathrm{Sr}$ source.

\section{Single-event spectra}

A low dose rate ${ }^{241} \mathrm{Am}$ source $(3.5 \mathrm{MBq})$ was used for recording single-event spectra by means of conventional pulse measurement with one of the detectors. The main component of the ${ }^{241} \mathrm{Am}$ spectrum is the $60 \mathrm{keV} \gamma$-peak; the $\alpha$-radiation is absorbed within the source and its coverage. The distributions were measured down to imparted energies of $0.1 \mathrm{keV}$. Lower energies imparted could not be resolved due to insufficient signal-to-noise ratio. From the measurements the first and second moment of energy imparted per event were obtained:

$$
\bar{\varepsilon}=1.64 \mathrm{keV} \quad \overline{\varepsilon^{2}}=6.47 \mathrm{keV}^{2}
$$

accordingly one has the weighted mean:

$$
\bar{\varepsilon}_{D}=\overline{\varepsilon^{2}} / \bar{\varepsilon}=3.94 \mathrm{keV} .
$$

The weighted mean of the specific energy is equal to the weighted mean of the energy imparted divided by the mass of the sensitive volume.

\section{Control measurements}

In the determination of microdosimetric parameters one must ensure that the influence of signal noise, of offset currents, and of other electronical artefacts 
does not add appreciably to the observed variance of the signals. The influence of the different factors was, therefore, investigated in control experiments performed without radiation field but with, otherwise, equal conditions.

Determining chains of voltage values with different sampling frequencies one obtains a spectrum of differences between successive values with a mean close to zero and with a standard deviation of $\sigma_{a}=20 \mu \mathrm{V}$. This value is substantially independent of gas pressure, detector voltage, and sampling frequency within the normal operating range. The main contribution to the observed variance is due to the electronic noise of the electrometers. Any noise increases the measured value of $\bar{\varepsilon}_{D}$, because it is uncorrelated in the two detectors; while it increases the variance in each detector it does not contribute to the covariance. The ratio of noise to variance increases with decreasing absorbed dose per sampling interval, and this puts certain constraints on the required dose rate or the permitted sampling frequency. Hence, the parameters must be chosen so that the dose per sampling interval exceeds a critical value. In the subsequent section, data are presented that were obtained under the condition that the ratio of the signal variance to noise variance was in excess of 10 .

Offset currents can be another critical factor, because they give rise to uncertainties in the variances which depend on the ratio of their magnitude to the magnitude of the detector current $I(\sim \dot{D}(t))$. The offset currents of roughly $10^{-14} \mathrm{~A}$ were sufficiently low in the present experiments to be of little concern even at the lowest dose rates employed.

\section{Variance-covariance measurements in constant fields}

3.1. Americium 241. Measurements were performed with the ${ }^{241} \mathrm{Am}$ source $(3.5 \mathrm{MBq})$ at a source-to-detector distance that resulted in a measured dose rate of $4 \mathrm{nGy} / \mathrm{s}$. Sampling rates of $1 \mathrm{~Hz}, 10 \mathrm{~Hz}, 100 \mathrm{~Hz}$, and $1 \mathrm{kHz}$ were employed. Gas pressure and gas multiplication were the same as in the single-event measurements. At the dose rate of $4 \mathrm{nGy} / \mathrm{s}$ the mean detector current of $6 \cdot 10^{-13} \mathrm{~A}$ exceeds the offset current by a factor of about 50 .

It is a somewhat undesirable feature of the prototype experimental set-up that it permits only four different sampling frequencies. However, other sampling frequencies can be readily simulated by utilizing only one out of $n$ sampling values, i.e. by treating $n$ successive sampling steps as one sampling interval. This has been done to obtain the data shown in Fig. 6. The results are based on 4 sampling series obtained at the different sampling frequencies. Each of the sampling series contains 500 data pairs. The left hand point on each of the 4 parts of the curve in Fig. 6 gives the value $\bar{\varepsilon}_{D}$ obtained when all data pairs are utilized for the computation of the variance. The next point gives the value that results when only each second pair is utilized. The last point in each series gives the result when only 1 out of 9 data pairs is utilized, i.e. when 9 steps are combined into one sampling interval. The statistics has to get worse when one uses effectively only $500 / 9 \approx 55$ sampling intervals instead of 500; however, the data show that, even with these fairly low numbers of sampling values, acceptable estimates of $\bar{\varepsilon}_{D}$ are obtained. 


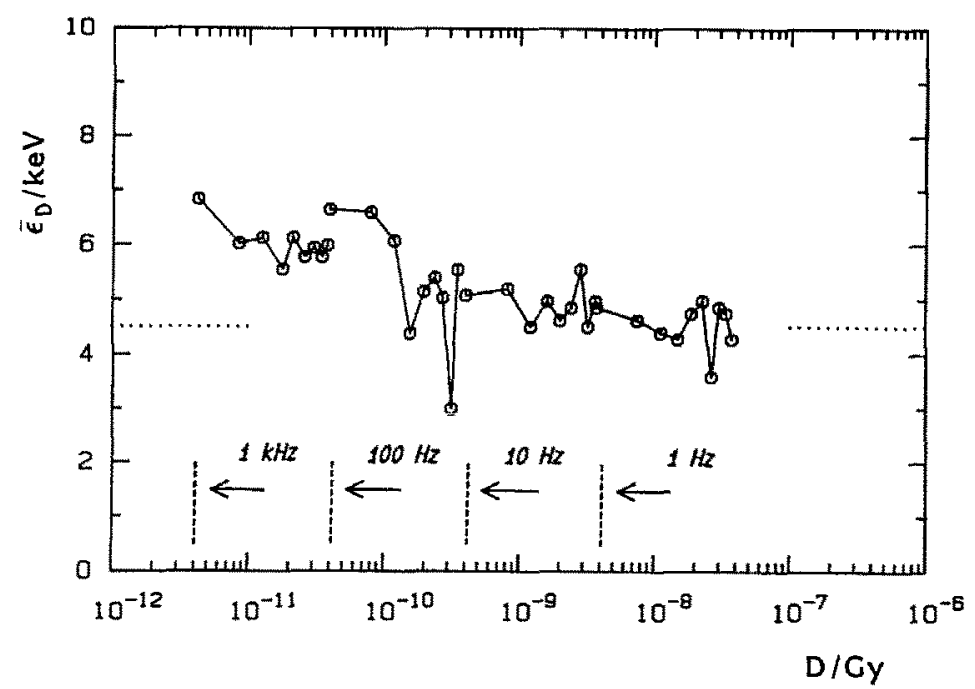

Fig. 6. Values of the dose mean energy imparted, $\bar{\varepsilon}_{D}$, obtained with different absorbed doses, $D$, from ${ }^{241} \mathrm{Am}$ per sampling interval. The dose rate was $4 \mathrm{nGy} / \mathrm{s}$. For four points the sampling frequencies are indicated; for details see text. The dotted line marks the value $\bar{\varepsilon}_{D}=4.5 \mathrm{keV}$ which is in accord with the data obtained at low and intermediate sampling frequencies

The results show the decreasing trend of the estimated value, $\bar{\varepsilon}_{D}$, with decreasing sampling frequencies, i.e., with larger doses per sampling interval. The broken line indicates a visual fit to the constant value that is reached at doses per sampling interval in excess of $1 \mathrm{nGy}(\Delta t>0.2 \mathrm{~s})$ :

$$
\bar{\varepsilon}_{D}=(4.5 \pm 0.5) \mathrm{keV}
$$

For sampling intervals shorter than $0.2 \mathrm{~s}$, i.e., dose increments less than $1 \mathrm{nGy}$, the sensitivity begins to be inadequate. Above this level the ratio of the signal variance to the noise variance is sufficient, and there is no recognizable bias. The deduced minimal absorbed dose per sampling interval of about $1 \mathrm{nGy}$ corresponds to about 5 energy deposition events, and one can, therefore, conclude that the present method is applicable under the condition that the majority of sampling intervals experience at least 1 energy deposition event. Maximum doses per sampling interval have not been employed in this preliminary study; however, they can be estimated as follows. The dynamic range of the ADCs is $10 \mathrm{~V}$. To have at least 10 sampling intervals in a cycle, the mean voltage difference must not exceed $1 \mathrm{~V}$, which corresponds to $1.5 \mu \mathrm{Gy}$ without amplification by the main amplifier and with the chosen operational conditions of the detectors. In the present experiments one has, therefore, an operating range of about:

$$
1 \mathrm{nGy}<D<1 \mu \mathrm{Gy} \text {. }
$$

In a given radiation field the doses per sampling interval may, of course, be constrained by the magnitude of the necessary time intervals. If the duration 


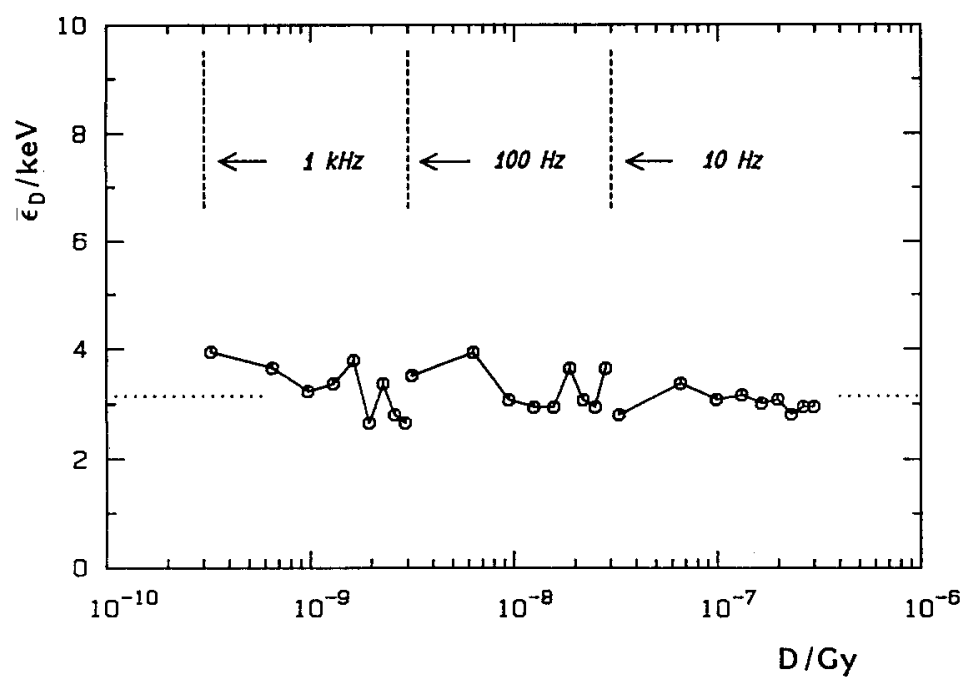

Fig. 7. Values of the dose mean energy imparted, $\bar{\varepsilon}_{D}$, obtained with different absorbed doses, $D$, from ${ }^{90} \mathrm{Sr}$ per sampling interval. The dose rate was $300 \mathrm{nGy} / \mathrm{s}$. For three points the sampling frequencies are indicated; for details see text. The dotted line marks the value $\bar{\varepsilon}_{D}=3.14 \mathrm{keV}$

of the sampling intervals becomes to long - and in the present experimental configuration the limit may be about $10 \mathrm{~s}$ - voltage shifts and uncontrollable offset currents may falsify the measurements.

Measurements are feasible up to a dose rate which is determined by the maximum dose per sampling interval and the maximum sampling frequency. The maximum dose of $1 \mu \mathrm{Gy}$ and the maximum sampling frequency of $1 \mathrm{kHz}$ correspond to the dose rate $1 \mathrm{mGy} / \mathrm{s}$. On the other extreme, measurements of $1 \mathrm{nGy} / \mathrm{s}$ - corresponding to a few energy depositon events per second - are feasible. One concludes that this experimental configuration can be employed over a remarkable range of 6 orders of magnitude in dose rate. Further expansion of this range can be achieved by adapting the gas multiplication and/or the capacity of the charge integrating capacitors to the field intensity.

The time required for recording a sampling series is a matter of seconds or minutes. Data processing and parameter evaluation is readily performed, so that the result of one measurement is available within a few minutes (and this can, in principle, be readily accelerated).

3.2. Strontium 90. Similar investigations, as described above, have been performed with a ${ }^{90} \mathrm{Sr}$ source of $33 \mathrm{MBq}$, i.e. a $\beta$-emitter $\left(E_{\max }=0.55 \mathrm{MeV}\right)$, at a distance corresponding to $300 \mathrm{nGy} / \mathrm{s}$. Figure 7 shows the values of $\bar{\varepsilon}_{D}$ derived at different sampling frequencies, i.e., different values of absorbed dose per sampling interval. Utilizing the total dynamic range, one obtains a sufficiently broad plateau of constant values for $\bar{\varepsilon}_{D}$. At fixed dose rate the absorbed dose per sampling interval can cover three orders of magnitude $(1 \mathrm{~ms}<\Delta t<1 \mathrm{~s})$. The minimal absorbed dose is determined by the condition that the ratio of the 
signal variance to noise variance be approximately 10 . Hence, the maximum applicable sampling frequency is about $100 \mathrm{~Hz}$, corresponding to a minimal dose of $2 \mathrm{nGy}$. As indicated in Fig. 7 the weighted mean energy imparted is:

$$
\bar{\varepsilon}_{D}=(3.14 \pm 0.4) \mathrm{keV} \text {. }
$$

\section{Varying radiation fields}

In the experiments with constant dose rate, the ratio of the relative variance and the relative covariance was 10 or more, i.e., the uncorrelated variations were dominant. To assess its efficiency, the method needs to be employed at ratios, $V / C$, of the order of 1., i.e., under conditions where the correlated variations contribute a substantial, or even the predominant part of the variance. This condition can apply in time-varying radiation fields, and one must examine the precision of the measurements which can then be attained.

Figure 8 shows the cumulative signals (specific energies in the detectors corresponding to the integrated currents) of the two detectors irradiated by the ${ }^{90} \mathrm{Sr}$ source. Successive cycles have been joined for the diagram. The dose-rate variations were produced by inserting, in an irregular time pattern, a thin absorber between source and the pair of detectors. The resulting changes of dose rate are readily recognized in the parallel waves of the two signals of cumulated specific energy and are equally apparent in the increments of specific energy in the lower panels of the figure. The corresponding correlation diagram is shown in Fig. 9. The ratios $V_{A} / C_{A B}$ and $V_{B} / C_{A B}$ are roughly 1.7. From this
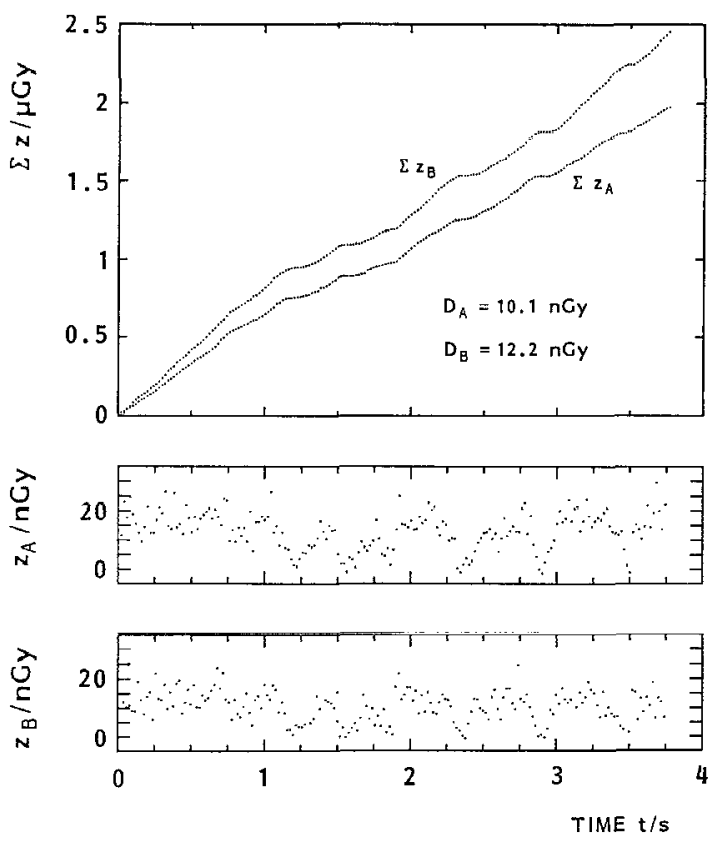

Fig. 8. Time-course of the cumulated specific energy, $\Sigma z$, in a time-varying radiation field of a ${ }^{90} \mathrm{Sr} \beta$-emitter. The sampling rate was $f=50 \mathrm{~Hz}(\Delta t=20 \mathrm{~ms})$, the total number of sampling points $N=188$. The mean values, $\bar{z}=D$, are indicated. The corresponding mean energies imparted are $\bar{\delta}_{A}$ $=29.9 \mathrm{keV}$ and $\bar{\varepsilon}_{B}=36.2 \mathrm{keV}$. In the two lower panels the corresponding increments of the specific energies are shown for the two detectors 
Fig. 9. Correlation diagram of the increments of the specific energies, $z_{j}$, in the detectors $A$ and $B$. The dashed lines represent the mean values $\bar{z}=D$. The relative variances, $V$, and the relative covariance, $C_{A B}$, are indicated

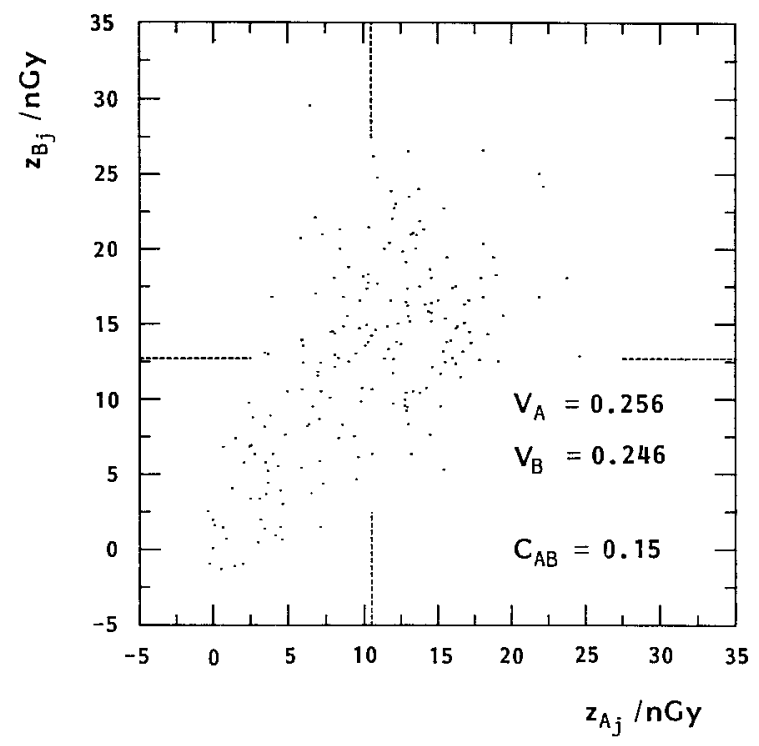

single sampling series $(f=50 \mathrm{~Hz}, N=188)$ one derives the following values of the dose mean energy imparted per event:

$$
\begin{aligned}
& \bar{\varepsilon}_{D}=\left(V_{A}-C_{A B}\right) \cdot \bar{\varepsilon}_{A}=(0.256-0.15) \cdot 29.9 \mathrm{keV}=3.17 \mathrm{keV} \\
& \bar{\varepsilon}_{D}=\left(V_{B}-C_{A B}\right) \cdot \bar{\varepsilon}_{B}=(0.246-0.15) \cdot 36.2 \mathrm{keV}=3.47 \mathrm{keV}
\end{aligned}
$$

This is consistent with the result obtained at constant dose rate.

In Fig. 8 the ordinate values are given in terms of the actual specific energy in the sensitive regions of the detectors. This is a somewhat unusual procedure because specific energy is commonly related to the simulated site (assumed density: $1 \mathrm{~g} / \mathrm{cm}^{3}$ ) where its value is higher by a factor that equals the square of the ratio of densities $\left(1.78 \cdot 10^{8}\right.$ in these experiments). It is, however, felt that the close relation between specific energy and actual absorbed dose should be preserved in the present context, to indicate more clearly that the measurement can be performed in radiation fields of extremely low dose rate. To obtain the microdosimetric parameter $\bar{z}_{D}$ one needs to devide $\bar{\varepsilon}_{D}$ by the mass of the simulated site; In the present example this results in $0.19 \mathrm{~Gy}$ and $0.21 \mathrm{~Gy}$.

\section{Discussion}

The preliminary applications and results which are here presented illustrate some of the potential of the method. Over a wide range of doses and dose rates it permits the determination of weighted mean values of lineal energy, specific energy, or energy imparted.

The twin-detector method can be seen as a generalization of the variance method which has been introduced by Bengtsson et al. (Bengtsson 1970; Bengtsson and Lindborg 1974). For this earlier method various detector systems have 
been utilized. Forsberg and Lindborg (1981) employed a modification of a parallel-plate ionization chamber which made it possible to simulate very small regions by the utilization of low voltages and low pressures. Rikner et al. (1984) applied semi-conductor detectors which have the advantage of small sensitive volume and of correspondingly high spatial resolution. The authors specified the gain of their detectors in terms of a dose sensitivity, i.e., detector charge per unit absorbed dose, of $40 \mathrm{nC} / \mathrm{Gy}$; this agrees roughly to the corresponding parameter in our experiments.

Lindborg et al. (1984) were the first to develop and employ a twin-detector device. It consisted of two uncoupled counters, housed in a single thimble-shaped outer shell of tissue equivalent plastic. The configuration was not a prototype, and permitted practical measurements and easy handling of the twin-detector due to the integrated construction of the counters.

In the studies of Lindborg et al. $(1984,1985)$ electrometers were used in the current-amplification mode. A drawback of their utilization of current-reading electrometers are the unavoidably longer time constants. Integration times down to $0.1 \mathrm{~s}$ ( 5 time constants) have been used. The time constant is proportional to the feedback resistance which at higher dose rates may have to be lowered. Charge amplifiers permit sampling intervals down to $1 \mathrm{~ms}$ or less. A somewhat unattractive feature of the method is that the charge capacitors must be discharged periodically. This might lead to certain complications, if the registration of the signals needs to be phased to periodicities of pulsed fields. However, the intermittent measurement with periodic brief pulses will generally pose no major problem.

Kliauga et al. (1986) used the variance-covariance method for measurements on a therapy beam from a pulsed linear accelerator and reported measurements for $10 \mathrm{MeV} \mathrm{x}$-rays and 9 and $18 \mathrm{MeV}$ electrons. In their system they triggered the current-reading electrometers according to the pulse rate of $360 \mathrm{~Hz}$ of the accelerator, obtaining in this way one pair of data per machine pulse. With our charge reading instrumentation similar triggering could be employed. However, measurements could also be performed with a sampling rate which is uncorrelated. In either mode of operation one would have the advantage that no pulse-shaping techniques are required.

The registration, transfer, and processing of the data requires only conventional procedures and does not limit the precision or sensitivity of the method. In the practical implementation, staggered data transfer along a single channel may be desirable. Simple methods of signal delay would be sufficient to achieve proper multiplexing.

The general advantages of the method have been pointed out when it was originally introduced (Kellerer and Rossi 1984) and have been demonstrated in the applications to time-varying fields and to pulsed accelerators for clinical use (Kliauga et al. 1986). The present results show the sensitivity of the method that can be attained in the current-integrating mode in fields of low dose rate, and they demonstrate the applicability to a broad variety of irradiation conditions. 


\section{References}

Bengtsson LG (1970) Assessment of dose equivalent from fluctuation of energy depositions. Proceedings of the Second Symposium on Microdosimetry, EUR-4552:375 400

Bengtsson LG, Lindborg L (1974) Comparison of pulse height analysis and variance measurements of the determination of dose mean specific energy. Proceedings of the Fourth Symposium on Microdosimetry. EUR-5122:832-842

Forsberg B, Lindborg L (1981) Experimental limitations in microdosimetry measurements using the variance technique. Radiat Environ Biophys 19:125-135

ICRU Report 33 (1980) Radiation quantities and units. International Commission on Radiation Units and Measurements, Washington, D.C.

ICRU Report 36 (1983) Microdosimetry. International Commission on Radiation Units and Measurements. Bethesda, Maryland

Kellerer AM, Rossi HH (1984) On the determination of microdosimetric parameters in timevarying radiation fields: the variance-covariance method. Radiat Res 97:237-245

Kliauga P, Amols H, Lindborg L (1986) Microdosimetry of pulsed radiation fields employing the variance method. Radiat Res 105:129-137

Lindborg L, Kliauga P, Marino S, Rossi HH (1984) The variance-covariance method: electric current measurements. DOE Annual Report, Columbia University

Lindborg L, Kliauga P, Marino S, Rossi HH (1985) Variance-covariance measurements of the dose mean lineal energy in a neutron beam. Radiat Prot Dos vol 13, nos 1-4:347--351

Rikner G, Grusell E, Hogstrom B, Jung B, Maripuu E (1984) Variance measurements with two semiconductor dose detectors. Acta Radiol Oncol 23:471-475 